\title{
Robotic Extramucosal Excision of Bladder Wall Leiomyoma
}

\author{
Khalid E. Al-Othman, Emad S. Rajih, Mohammed F. Al-Otaibi \\ King Faisal Specialist Hospital \& Research Center, Riyadh, Saudi Arabia
}

\section{ABSTRACT}

Introduction: Multiple case reports and reviews have been described in the literature for bladder wall leiomyoma resection via different approaches. The minimally invasive partial cystectomy remains the most widely accepted technique; however, case reports for enucleation of bladder wall leiomyoma have also been described. The purpose of this video is to demonstrate the robotic extramucosal excision of a bladder wall leiomyoma, without cystotomy, but with complete removal of the muscular layer.

Materials and Methods: A 35-year old male present with lower urinary tract symptoms and imaging showed bladder wall mass with histopathology showed leiomyoma. The patient consented for mass excision with the possibility of a partial cystectomy. The patient was placed in the supine, 30-degree Trendelenburg position during the procedure. A total of 4 ports were inserted. A 3-arm da Vinci robotic surgical system was docked, and the arms were connected. Extramucosal excision was accomplished without cystotomy and muscle approximation was achieved by 20 Vicryle.

Result: The operative time was 90 minutes, blood loss of approximately $50 \mathrm{~mL}$ and the patient was discharged after 72 hours with no immediate complications and a 6 months follow-up showed no recurrence.

Conclusion: Such a technique results in complete excision of the tumor, without cystotomy, and also maintains an intact mucosa. These steps, in addition to decreasing the risk of local recurrence, also shorten the period of postoperative catheterization and hospitalization.

\section{ARTICLE INFO}

Available at: www.brazjurol.com.br/videos/january_february_2014/Al-0thman_127_128video.htm

Int Braz J Urol. 2014; 40 (Video \#3): 127-8

Submitted for publication:

December 01, 2013

Accepted after revision:

January 30, 2014
Correspondence address:

Khalid Ebrahim Al-Othman, MD Associate Professor uro-oncologist Medical and Clinical Affairs MBC 86 King Faisal Specialist Hospital \& Research Center P.O.BOX 3354, Riyadh 11211, Saudi Arabia Fax: 009661 442-4491 E-mail: kialothman@gmail.com 


\section{EDITORIAL COMMENT}

Al-0thman and colleagues from Riyadh Saudi Arabia present an elegant video demonstrating the robotic excision of a bladder leiomy- oma. This is a rare, but good example of how the robot can be used to perform what in essence is a partial cystectomy. The visualization was perfect, the dissection meticulous and the reconstruction was beyond reproach.

A. Karim Kader, $M D$

Associate Professor

Department of Urology

University of San Diego San Diego, CA, USA

E-mail:jmthompson@ucsd.edu 